\title{
Cellular and Molecular Bioengineering: A New Editorial Perspective
}

It is a great honor and privilege to have been selected as the next Editor-in-Chief of Cellular and Molecular Bioengineering. It is my opinion, and the opinion of everyone I have talked with within the biomedical engineering community, that the journal is built on a solid foundation with a great potential for future growth and success as a venue to communicate high quality research in cellular and molecular bioengineering. For this we owe a great debt and I extend my sincere, personal thanks to the previous Editors, Ed Guo and David Odde, the Editorial Advisory Board of the journal, as well as the Executive Committee of the Biomedical Engineering Society, for their work and foresight in establishing this journal. As we set out on the task of leading CMBE to even greater success, and thus serve the membership of the Biomedical Engineering Society, I would like to take this opportunity to share with the readership my vision for the future of this journal.

The CMBE journal has consistently published high quality, fundamental studies into molecular and cellular scale phenomena, as evidenced by previous special issues on cell mechanics, mechanobiology, and molecular motors to name a few. In addition to publishing these important fundamental studies, I would like also to feature papers that emphasize the " $M$ " in BMES, for instance the clinical and translational applications that motivate much of our work in biology. Bioengineers have made important scientific contributions in many subdisciplines that mechanistically bridge between the molecular and cellular scales. One example of this is macromolecule-conjugated nanoparticles designed to elicit biological responses at the cellular level. Another example is the development of cell-based systems biology models, many of which can now be coupled to spatially resolved modules that account for the diffusive transport of reacting species and cytoskeletal mechanics. To better emphasize that CMBE is a well-suited publishing home for these different types of papers, we will be reorganizing the journal into different Sections. Some of the Sections are already well represented within the existing volumes of CMBE: Cellular; Molecular; Mechanobiology; Electrophysiology. Other Sections will represent a broadening of the current scope, while still residing within the overarching theme of engineering studies that bridge the molecular and cellular scales: Nanomedicine; Systems Biology; Clinical \& Translational.
We will reach out to potential authors in related cell biology and clinical communities, continue to work closely with the Cellular and Molecular Bioengineering Special Interest Group of BMES, and feature occasional Special Issues devoted to emerging topics important to the CMBE readership.

We are currently reappointing a group of distinguished and active Associate Editors, who share in a commitment to build CMBE into an even stronger publication for the BMES community. Our editorial board will represent scientific, geographic, and demographic diversity that covers the spectrum of cellular and molecular bioengineering. I am also a strong believer in the value of social media in propelling human interactions in scientific research. To keep abreast of the exciting new developments at CMBE in the coming months and years, I encourage you to follow us on Twitter (@CMBEjournal) and like us on Facebook (www.facebook.com/CMBEjournal). We have a number of innovative new ideas in the works to increase the visibility of papers once they are published in CMBE, and these will begin to be implemented over the next year.

As our email inboxes can readily attest, researchers have a widening array of publishing options and conferences to attend, some more worthwhile than others. As an official journal of the Biomedical Engineering Society, CMBE offers a place where your research will be reviewed, noticed, and read by your peers. So join us as we embark on a journey to take the journal to ever greater scientific reach and impact. Submit your best work (!) that applies tools and concepts from engineering to solve important problems in biology, particularly at the cellular and molecular scales. And finally, your feedback is always welcome, whether you see me at the BMES Annual Meeting, reach me by phone (607-255-9803) or contact me online. Enjoy this June issue, which features a group of papers designated as Outstanding Contributions from the 2012 BMES Meeting, as well as an excellent collection of regular articles.

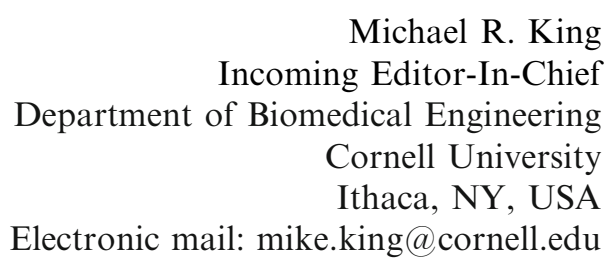

Michael R. King Incoming Editor-In-Chief Cornell University Ithaca, NY, USA Electronic mail: mike.king@cornell.edu 